\title{
PRIAPUS - non-contact monitoring of penile dimension changes by photogrammetry
}

\author{
W.F. Thon ${ }^{1}$, C.G. Stief ${ }^{1}$, A.E. J. L. Kramer ${ }^{1}$, F. Leberl ${ }^{2}$, and U. Jonas ${ }^{1}$ \\ ${ }^{1}$ Department of Urology, Hannover Medical School, Konstanty-Gutschow-Strasse 8, D-3000 Hannover 61, Federal Republic of Germany \\ ${ }^{2}$ Vexcel Corporation, Boulder, CO, USA
}

\begin{abstract}
Summary. Clinical evaluations to objectify sexual arousal in men use indicators of penile configuration changes. Most common are measurements of changes in circumference and/or rigidity using loops around the penis. To avoid psychogenic disturbance during the investigation, a method was developed that enables non-contact evaluation of penile changes during visual sexual stimulation. A three-dimensional (3-D) real-time computerized imaging system is used for the acquisition and registration of penile configuration changes. Images from two high-precision cameras are digitized using a geometrical and graylevel registration model. The triangulation technique in the stereoscopic approach is used to extract 3-D information. This method of digitized photogrammetry enables non-touch documentation of penile length, diameter, volume, curvature and direction changes during sexual stimulation. The curvature registration also enables objective documentation in patients complaining of penile curvature during erection.
\end{abstract}

The evaluation of penile erectile dysfunction has progressed over the last 15 years. New diagnostic procedures have been established to provide accurate and less invasive means of determining the etiology of the disorder [4]. Monitoring of erectile responses to erotic visual stimuli, initiated and mediated by cognitive factors, is preferred to nocturnal tumescence and rigidity measurements. To avoid psychogenic disturbances during investigation, a non-contact device for recording of penile configuration changes is regarded as being the ideal method.

In patients complaining about penile curvature during erection, the physician usually performs a clinical inspection of the patient's erect penis or judges autodocumentation photographs. The use of photographic documentation is limited, as quantitative parameters are not easily derived.

At present, no device exists for objective monitoring and documentation of penile erection or configuration abnormalities by a non-contact method. In this paper the preliminary results obtained using an automatic image-processing unit for penile evaluation in patients with erectile disorders are described.

\section{Materials and methods}

The PRIAPUS system consists of two major units, the recording and the processing unit. The recording unit collects a stereo pair of images from two CCD video cameras (Pulnix TM 540), each with a $512 \times 512$-pixel array, a field of view of about $200 \times 200 \mathrm{~mm}^{2}$ and a field depth of about $300 \mathrm{~mm}$. The processing unit consists of a Unix work station, a Sun 386i desktop microcomputer with graphic display and an image-processing subsystem.

This image-processing subsystem (Matrox framegrabber) accepts video signals from the cameras of the recording unit and converts them to digital image data. The grey levels of these images are processed [3] to deliver a binary view: all grey values above the threshold are replaced by a fully saturated (white) representation; below the threshold the grey levels are replaced by zero saturation (black). Each object in the field of view, at this point defined in a complete black-and-white sense, is stored as a boundary outline: the objects are represented by their silhouettes. The silhouette outlines in the frame storage are used to determine the measurements. A calibration procedure is necessary for calculation of the video cameras' orientation. This calibration is performed separately with the help of a three-dimensional (3-D) calibration toolbox.

A disposable sheet (white for dark-tanned men and black for those with light skin) provides the image with sufficient contrast that the penis is clearly detectable and separate from the background. At a minimal sample time of once every $15 \mathrm{~s}$, the computer automatically collects a stereo pair of images and processes them to pairs of outlines for storage.

After the entire patient session has been finished, the outline silhouettes are available for analysis. The analysis 

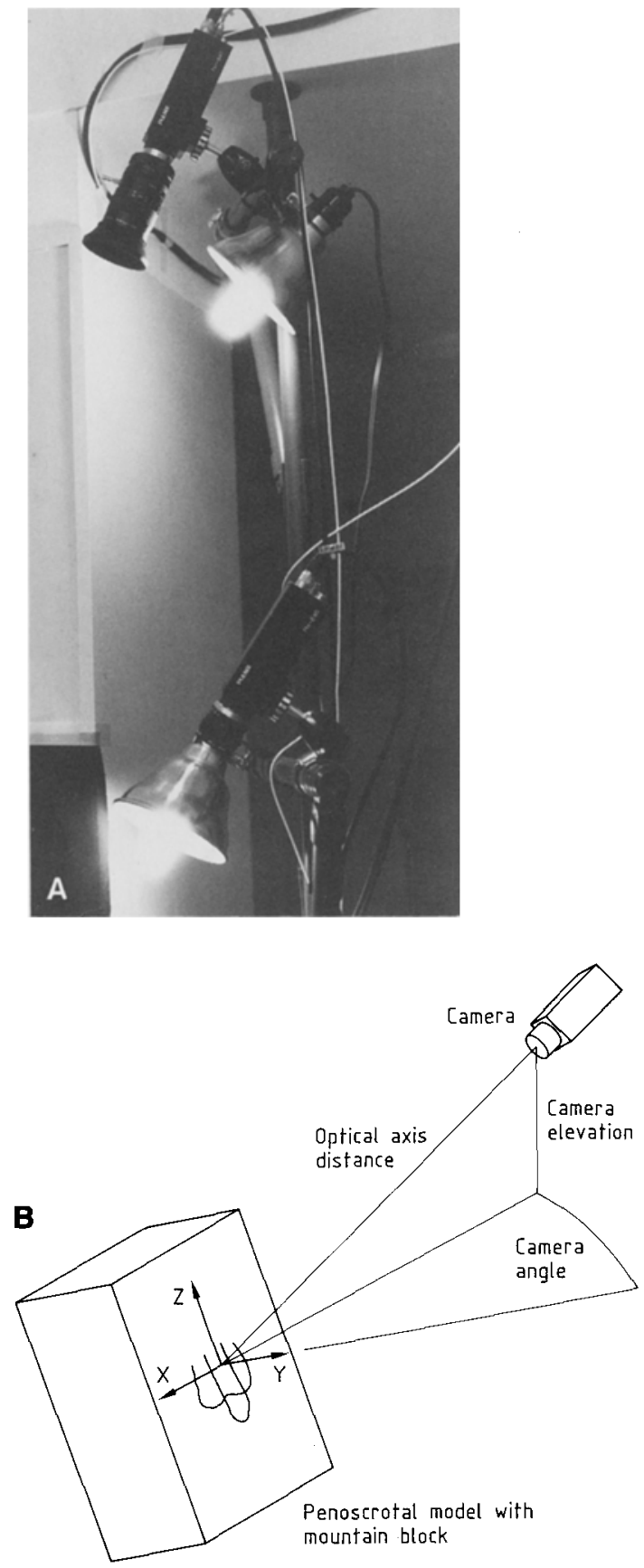

Fig. 1. A Position of the two video cameras with incandescent lamps in the investigation room. B Definition of camera distance, angle and elevation

sequence can automatically select the largest object in the field of view as being the penis and defines its basis as the reference point in space. Alternatively, the penile basis can be marked interactively by the investigator. Stereocorrelation establishes the camera-to-organ distance and the cameras' angles and elevation (Fig. 1) relative to the base and at the tip of the penis, defining the extremities by means of triangulation processing $[1,2]$.

The post-processing includes slicing of the outline of one image of the penis into 30-50 discs (Fig. 2). Volume
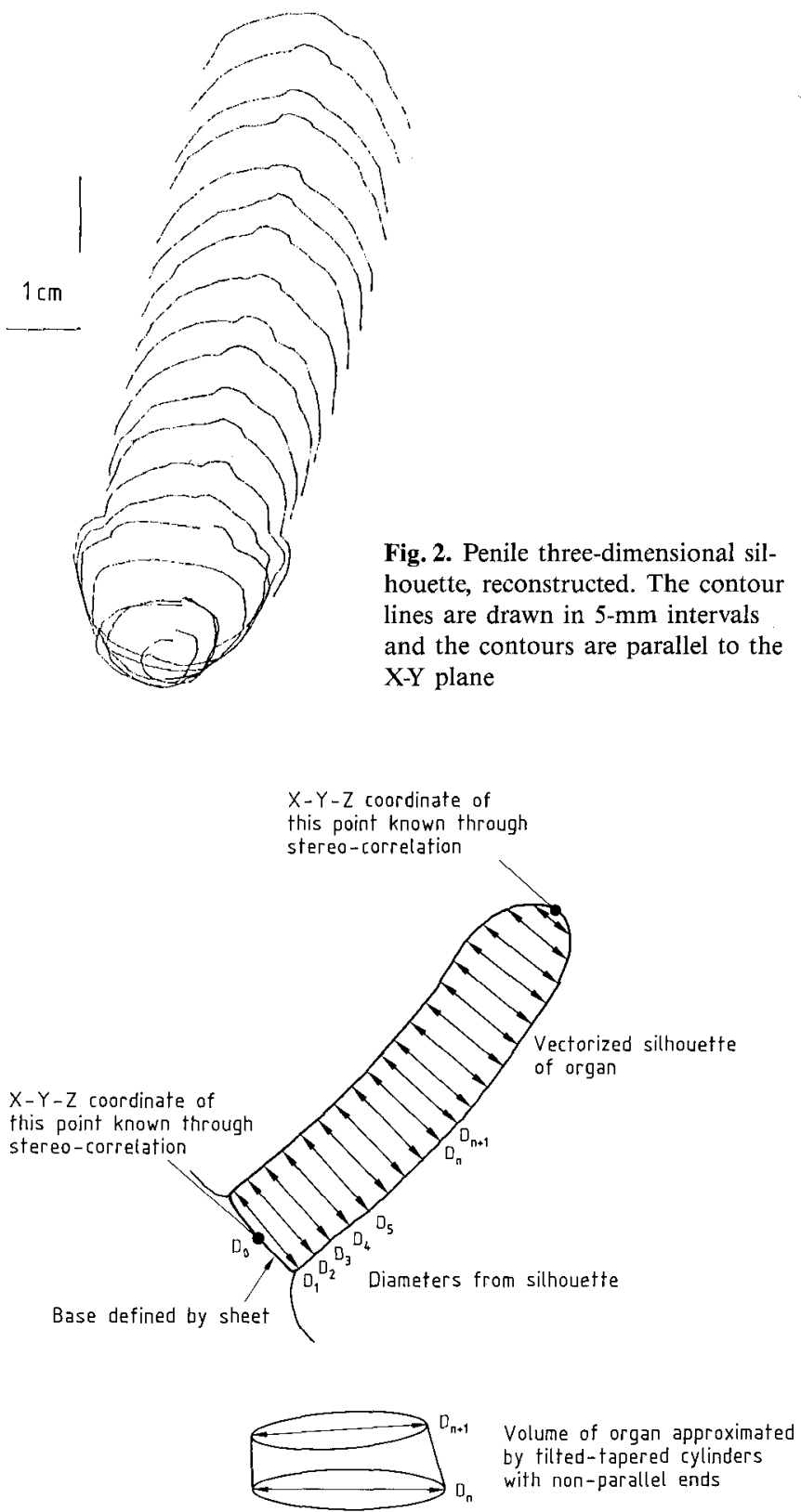

Fig. 3. Illustration of length and volume measurements

is calculated by summing the volumes of all discs (Fig. 3). The system-user interface presents a number of display screens for choosing different types of information. Evaluation data are displayed against time: length, average and maximal diameter, angle of base to tip off horizontal, curvature per centimeter of length, and volume of the organ (Table 1). The maximal bending angle and its location on the penis, as well as the location of the maximal diameter, are also available in the data files. The views of the penis (shapes) from both cameras can be displayed as well.

For test measurements a penoscrotal model was used. After the environmental conditions of the system had been established, mainly the camera position, volunteers without erectile disorders and patients with erectile dys- 
Table 1. Calculated data in the PRIAPUS recording

1. Penile length

2. Average penile diameter

3. Maximal penile diameter

4. Angle of base to tip off horizontal

5. Curvature per $\mathrm{cm}$ length

6. Penile volume

7. Maximal bending angle

8. Location of maximal bending angle

9. Location of maximal diameter

Graphs of the trend vs time are available for the data 1-6

functions were investigated. All men were seated and were exposed to erotic visual stimulation. In patients, intracavernous injection of a vasoactive drug (papaverinephentolamine combination) was performed to induce an erection.

\section{Results}

A pilot study demonstrated that two cameras are needed for accurate measurements; the penile movements from the flaccid state to erection and back are not confined within a single plane; thus two-dimensional measurements through a single camera do not lead to a reliable documentation of penile configuration changes. The test measurements obtained using the 3-D system of length, angle and volume in the penoscrotal model and in patients lead to several conclusions.

The appropriate camera position should be more to the side of the penis, as the major direction of movement of the penis in the sitting position is more or less vertical. The correct definition of the penile base is important, as the analysis software keeps the penile basis as a reference point throughout the calculations and tries to follow this point in the case of patient movement. The volume calculation must be based on the true silhouette, i.e., on a more ellipsoidal cross section defined by the diameters of both stereo measurements. Penile angle computations should be based on regression lines rather than on lines through beginning and end points.

In stereophotogrammetry, the intersection angle between the two cameras must be carefully considered. Small intersection angles in the lines of view result in large errors in the measurement of the third dimension. Intersection angles that are too large result in an inability to match the paired images.

Large camera-to-object distances prove to be impractical in terms of the confines of the examination room and the accuracy of the measurements. Accuracy increases through an increase in resolution as the camera gets closer to the penis. Camera positions too close to the patient affect the response psychogenically.

Our results in 5 volunteers show that during the beginning of the tumescence phase, the penile diameter, length and volume increase in a coordinated way, with no major penile straightening or lifting from its resting position. The fully rigid erection phase is characterized by such straightening and lifting; however, these are marked by only minimal changes in the dimensions of the organ, which by then is completely filled with blood.

\section{Discussion}

A prototype system was developed that aids the urologist in diagnosing erectile disorders. The advantage of the PRIAPUS system as compared with existing products on the market in the evaluation of erectile dysfunction is its physical non-contact evaluation. The system is capable of making detailed measurements of circumference, penile shape, length, volume, curvature and erection angle. It enables the objective determination of penile configuration in patients complaining about bending in the erect state. The system's accuracy in indirectly measuring the rigidity and calculating volume changes and angle changes over time should be determined in further investigations.

The device uses computerized image processing and photogrammetric techniques to measure penile configuration from two video cameras' stereo images. The number of image pixels has a direct bearing on the accuracy of the system. The fewer the number of pixels making up the image, the less accurate it becomes. Accuracy is also a function of the exactness of the calculated organto-camera distance. A $1 \%$ error translates directly to a $1 \%$ error in length and diameter, but it corresponds to a $3 \%$ error in the calculation of the volume.

Test measurements in a penile model and in men lead to the conclusion that both cameras need to be on the same side of the penis, with a minimal angle of about $45^{\circ}$ and a maximal angle of about $75^{\circ}$. Although the measurements of penile length and volume do not measure the crural area, they are an accurate measurement of the important part of the penis involved in erectile changes. Due to contrast processing, the lighting must be constant; changing of environmental lighting also changes the grey values in the camera images. The investigation therefore takes place in a darkened room and the penile area is lighted by incandescent lamps.

The preliminary results demonstrate that stereo measurements of the flaccid and erect penis are feasible. This supports the expectation that the PRIAPUS evaluation system can be integrated into the diagnosis of erectile dysfunction.

Acknowledgement. This investigation was supported by FM Wiest and Co. (Unterhaching, FRG) and Vexcel Corporation (Boulder, Co., USA).

\section{References}

1. Albertz J (1986) Digitale Bildverarbeitung in der Nahphotogrammetrie - neue Möglichkeiten und Aufgaben. Bildmess Luftbildwesen 54:34-45

2. Gruen A (1988) Towards real-time photogrammetry. Photogrammetria 42:209-244

3. Real RR (1982) Fast digital image enhancement for video image transfer in photogrammetric instrumentation. Int Arch Photogramm 24:201-212

4. Sidi AA, Lange PH (1986) Recent advances in the diagnosis and management of impotence. Urol Clin North Am 13:489-500 